\title{
ENFOQUE SISTÉMICO \\ EN LA FORMACIÓN PROFESIONAL \\ DEL AUDITOR COMO CAPITAL HUMANO
}

SYSTEMIC APPROACH IN VOCATIONAL TRAINING OF HUMAN CAPITAL AS AUDITOR

Félix Armando Rivera León*

[RECEPCIÓN: ABRIL 2016 / CONFORMIDAD: MAYO 2016]

\section{RESUMEN}

El objetivo de la investigación es el de analizar la capacitación y entrenamiento en estudios adicionales a la licenciatura especifica del profesional, y de otras especialidades de acuerdo a sus necesidades, que le permita tener un nivel de conocimiento para intervenir en la evaluación de la organización en estudio; así mismo, determinar la experiencia, como lo ético/moral, que se adquieren a través del dominio en contacto del día a día, en las áreas sustantivas de la organización de acuerdo a su objeto, materia de estudio, permitiendo contribuir al desarrollo de los atributos personales como capital humano.

Palabras clave: Sistémico; profesional; auditor.

\section{ABSTRACT}

This research is oriented analysis and training in additional studies to the degree specific professional, and other specialties according to your needs, enabling him to have a level of knowledge to intervene in the assessment of the organization under study; Likewise, assess the experience, as ethical / morality, which are acquired through the domain contact day by day in the substantive areas of the organization according to its object, subject matter, allowing contribute to the development of the personal attributes as human capital.

Keywords: Systemic; professional; auditor.

Doctor en Ciencias y Empresariales por la Universidad Nacional Mayor de San Marcos, Magister en Administración con mensión en Gestión Empresarial, Contador Público Colegiado, Profesor Principal de la Facultad de Ciencias AdministrativasUNMSM. 


\section{INTRODUCCIÓN}

Las nuevas tendencias en el mundo de la economía y del trabajo y la presencia dentro de las organizaciones de una tecnología cada vez más cambiante, suponen un reto progresivamente creciente para las empresas, que sólo las mejor preparadas podrán superar para asegurar su supervivencia. Repetidamente se ha comprobado, en ocasiones a través de experiencias dolorosas, que estar "mejor preparado" significa simple y llanamente, contar con un contingente humano integrado, sólidamente formado, motivado, comprometido, actuando coordinadamente y que aplique todo su potencial al logro de las políticas objetivos, metas y estrategias corporativas .

Es por ello que se ha detectado y comprobado, mediante la investigación realizada por el autor de la presente, que la formación del auditor, no tiene un modelo de formación actualizado y moderno, afectando de esta manera a la competitividad que debe tener el profesional de la especialidad.

De otro lado; las universidades no cuentan con un mecanismo adecuado para hacer un seguimiento efectivo de sus auditores egresados, generando con ello un vacío entre el profesional que hemos formado y la organización a ser evaluada.

La capacidad intelectual no es tan solo la clave, y en la nueva economía global de la información, es esencial los aspectos de experiencia profesional en la materia, como esencial es la vivencia del ser humano a través de su desarrollo ético y moral del individuo, ésta será la que lleve a dicho profesional a realizar el asesoramiento eficaz y eficiente orientando a las empresas al fin empresarial esperado. Hoy en día el talento humano importa mucho y va acompañado de la destreza y capacidad de acción de los recursos humanos que integran la organización.

Para la Administración Rodríguez, el fortalecimiento del capital humano debe ser enfocado desde cinco perspectivas: educación, salud, cultura, seguridad y género.

La educación es la que permite a los ciudadanos la inserción en trabajos mejor remunerados, además de generar una serie de aspectos que trascienden incluso a la forma de vida.

La salud es la que posibilita el bienestar físico de las personas, una de las características que ha distinguido a nuestro país en el mundo.

\footnotetext{
Plan de Desarrollo Humano........(Municipalidad de Hojancha), Costa Rica,
}

Aspiramos tener un ser humano integral, es por ello que el Plan Nacional de Desarrollo Humano ha considerado la cultura como uno de los pilares para fortalecer el capital humano del país.

Hay dos elementos en los cuales el país presentó en años anteriores algún tipo de deficiencias, específicamente en seguridad ciudadana y en la igualdad de género. Ambos elementos contribuyen a tener una sociedad más homogénea en la cual el desarrollo de los seres humanos facilite mejores condiciones de vida para todos los costarricenses.

Para la buena formación profesional de una persona intervienen una serie de factores, pero generalmente se da mayor énfasis a lo que son los conocimientos. Si hacemos un análisis veremos que la eficiencia y eficacia profesional se ve influida de alguna manera por otros factores, lo que hace que toda institución que forma profesionales debe conformar un equipo de especialistas que analicen esta realidad, para implementar correctivos a través del diseño de un modelo que resuma estas inquietudes. Idea fuerza que da lugar la presente investigación de un nuevo modelo a seguir.

En consecuencia el objeto de esta investigación es el lograr un enfoque en base a qué variables como es el conocimiento, la experiencia y lo ético / moral coadyuvan en la formación de un modelo profesional del auditor como capital humano de una organización y su actividad de inserción, con la eficiencia y eficacia profesional en el campo de la especialidad.

El estudio que se está presentando enfoca o establece los puntos de vista crítico actuales con respecto al enfoque a plantear (variables independientes) donde el Auditor de ahora, se forme mediante conocimientos vigentes y desarrollando habilidades que tiendan al talento creativo en el aspecto académico, de investigación y propios de la especialidad; de otro lado las empresas requieren asesores que orienten el uso y fomento de innovación tecnológica en la empresa y manejar o conocer el uso de los medios modernos de comunicación adecuados desde el punto de vista sistémico.

De otro lado ;debemos establecer las características y elementos que se van a considerar, que permitan alcanzar el éxito profesional a los auditores, en los diversos ámbitos de servicio (variable dependiente), donde el auditor aporta 
sus capacidades de creación, su experiencia para indicar pautas en el área económica / financiera, así como administrativa y sus actitudes de gestión en el accionar de actividades en las organizaciones, como son el campo de manejo de equipo, y administración, el de sistemas e informática y el de proyectos.

\section{FORMULACIÓN DEL PROBLEMA Problema general}

¿Cómo aplicar el enfoque sistémico en la formación profesional del auditor AdministrativoContable como capital humano?

\section{Problemas específicos}

1. ¿De qué manera la capacitación en estudios adicionales le permitan tener un nivel de conocimiento de la organización en estudio, constituyan y coadyuven al mejoramiento continuo del profesional especializado en auditoría?

2. ¿La experiencia profesional y el uso de recursos tecnológicos contribuyen al desarrollo de atributos personales del auditor Administrativo-Contable como profesional independiente?

\section{Justificación de la investigación}

La presente investigación basa su importancia y justificación en:

\section{Justificación teórica}

\section{Racionalización de la Administración}

La administración se debe racionalizar mediante un replanteamiento del proceso, y para ello; en primer lugar se debe capacitar al trabajador, a través de una cultura educacional, como es el control de calidad del producto y del servicio como filosofía que debe difundirse para lograr objetivos a mediano plazo.

En segundo lugar, se debe dotar de medios modernos para el manejo eficiente de la información en los diferentes niveles jerárquicos. Y en tercer lugar dotándoles de un presupuesto realista e incentivando a la obtención de recursos propios, pero con una reglamentación más flexible y que permita dinamizar la gestión empresarial.
Modernización y Profesionalización empresarial.

El profesional moderno debe estar a la medida de las circunstancias, pero para ello debe reunir ciertos requisitos obtenidos no solo en las aulas, sino de experiencias obtenidas en eventos académicos o a través de trabajos desarrolladas en la empresa.

Hoy en día la empresa necesita trabajadores bien preparados y con un grado de conocimientos que rebasan los niveles de primaria y secundaria.

\section{Apertura de nuevos sectores técnicos al trabajo profesional del Auditor.}

El auditor está muy relacionado al desarrollo de múltiples trabajos y de acuerdo a su formación accede fácilmente a campos laborales como la asesoría en la administración de empresas, logística empresarial, sistemas de información, industrias productivas en el sector comercial y en el de servicios generales.

Asimismo el auditor asume roles en el campo educativo, en el campo manufacturero y de servicio. De otro lado sirve como elemento de asesoramiento en el campo militar.

\section{Elevado nivel de competitividad profesional}

En la presente década aparece una corriente de competencia por obtener el liderazgo en la actividad que uno desarrolla, en este sentido, las empresas optan por obtener el trabajador más preparado y competente, para lo cual establecen una serie de requisitos que representan el tamiz que permitirá obtener los trabajadores que ellos desean, esto también se da por la cantidad de profesionales que ofrecen sus servicios pero que no están a la altura de los más competentes.

\section{Nivel significativo de trabajadores independientes}

Como producto del mayor incremento de la competencia las empresas optan por reemplazar el elemento humano por máquinas más eficientes, o en su defecto reducen al máximo sus costos siendo también el factor humano el que más se reduce, generando de esta manera desempleo, por lo que muchos de ellos con cierta experiencia en el ramo, crean su propia empresa y entran a competir en el mercado tanto de productos como de servicios, o también dedicándose al comercio ambulatorio, originándose por una u otra vía 
trabajadores independientes que se esmeran y se desarrollan ante las dificultades que enfrentan.

\section{Mayor competencia Universitaria}

Como producto de la competencia imperante en el ámbito empresarial, las organizaciones de formación profesional como son las Universidades realizan esfuerzos por formar profesionales cada vez más eficientes y eficaces, a través de nuevos currículos, mejores contenidos, docentes más capacitados y entrenados, y ofreciendo la mejor infraestructura con un apoyo excelente de medios y materiales educativos.

\section{Justificación práctica}

Incremento en el nivel de desempleo en el sector de mano de obra no tecnificada.

El análisis de la realidad nos indica que los más desprotegidos para obtener trabajo son los que tiene menos preparación en conocimientos educativos y técnicos, y como actualmente hay una mayor oferta de mano de obra, ello permite que los empresarios opten por escoger a los más preparados, utilizando personal con buen potencial en faenas rutinarias y no adecuadas para su nivel.

Los sectores de interés del país se centran en la minería, comercio, la industria, la agricultura y la educación.

El auditor en el Perú debe ser emprendedor para lograr que el país cambie su política y se dé un mayor realce y competencia al sector empresarial. Si bien es cierto que por nuestra geografía territorial los sectores de mayor interés para el país son la Minería, Agricultura y la Educación. El sector que en estos últimos años ha propiciado inversiones y generado más puestos de trabajo es el minero, pero es el que menos valor agregado genera al producto obtenido, desperdiciándose de esta manera posibles fuentes de trabajo y ganancias para el país.

Respecto a los sectores de Agricultura y Educación si bien es cierto son de interés para el país, pero el estado no adopta todavía políticas efectivas para lograr su crecimiento y desarrollo. Pues mientras al sector Agricultura no la tecnifique adecuadamente y le apoye a través de un financiamiento efectivo este sector no va despegar ni desarrollarse. Al sector educación le pasa algo similar pues requiere de una gran modernización en medio y materiales educativos y también de una filosofía educativa que se fije en los educadores y que ellos los transmitan a sus alumnos.

Por consiguiente, la presente investigación es importante para las Sociedades de Auditoría, Instituciones y Empresas en general ya que permitirá generar un marco laboral conceptual para delinear, en un futuro, estrategias de evaluación que conlleven a metodologías de formación profesional de los auditores, en todo su contexto integral.

\section{Limitaciones de la investigación}

Actualmente no existen metodologías que permitan una formación, en los aspectos de Auditoría que uniformicen criterios de formación. La formación profesional que al presente se realizan, se desarrollan sobre la base del criterio individual de las instituciones, el estudio se apoya en la experiencia profesional de los docentes; en lo que respecta al ámbito financiero se apuntalan en la utilización de las sociedades de auditoría, en cuanto a la aplicación y experiencias de realización en el mercado.

\section{OBJETIVOS DE LA INVESTIGACIÓN General}

Identificar la aplicación del enfoque sistémico en la formación profesional del auditor administrativo-contable como capital humano.

\section{Específicos}

1. Analizar que la capacitación en estudios adicionales, le permitan tener un nivel de conocimiento de la organización en estudio, constituyan y coadyuven al mejoramiento continuo del profesional especializado en auditoría

2. Evaluar si la experiencia profesional y el uso de recursos tecnológicos contribuyen al desarrollo de atributos personales del auditor administrativo-contable como profesional independiente.

\section{Hipótesis general:}

La formación profesional del Auditor Administrativo - Contable será eficaz si aplicamos un modelo que correlacione los factores de conocimientos, entrenamiento y lo ético-moral. 


\section{Hipótesis específicas:}

Primera hipótesis específica

Sólo el mejoramiento continuo del conocimiento del profesional especializado en auditoría le permitirá obtener un nivel de discernimiento para invertir en la evolución de organizaciones complejas.

\section{Segunda hipótesis específica}

La experiencia profesional, en lo ético /moral, el empleo puntual de diferentes recursos contribuyen al mejor rendimiento del proceso de aplicación en una auditoría.

\section{Identificación de Variables:}

Variable Independiente:

$\mathrm{X}=$ Formación profesional del auditor

Variable Interviniente:

$\mathrm{Y}=$ Mejoramiento continuo del conocimiento

Variable Dependiente:

$\mathrm{Z}=$ Experiencia profesional, ética /moral

\section{Operacionalización de las variables}

Indicadores de la Variable Independiente $X=$ Formación profesional del auditor

$\mathrm{X}_{1}$ Normas legales y contractuales

$\mathrm{X}_{2}$ Normas de Auditoría de Estados Financieros

$\mathrm{X}_{3}$ Planeación y Supervisión del Trabajo de Auditoría

$\mathrm{X}_{4}$ Control de Calidad de los Servicios de Auditoría

$\mathrm{X}_{5}$ Dictámenes de Estados Financieros

Indicadores de Variable Interviniente $Y=$ Mejoramiento continuo del conocimiento

$Y_{1}$ Pronunciamientos relativos a dictámenes NIIF's

$\mathrm{Y}_{2}$ Experiencias de aprendizajes en NIC's y

$\mathrm{Y}_{3}$ Estudios y evaluación del Control Interno

$\mathrm{Y}_{4}$ Muestreo Estadístico en Auditoría

$\mathrm{Y}_{5}$ Auditoría Operacional

Indicadores de Variable Dependiente $Z=$ Experiencia, ética moral, profesional del Auditor
$\mathrm{Z}_{1}$ Auditoría de Estados Financieros

$\mathrm{Z}_{2}$ Auditoría Interna

$\mathrm{Z}_{3}$ Auditoría Administrativa

$\mathrm{Z}_{4}$ Auditoría Integral

$\mathrm{Z}_{5}$ Auditoría

\section{Indicador general:}

El nivel de la auditoría y la calidad de su resultado, dependen de la preparación de conocimientos, como de su diligencia -experiencia; ético/moral- en tareas de evaluación en las organizaciones.

Las instituciones, cuya responsabilidad es la de formar profesionales, deben enfrentar el compromiso de que, ahí, el estudiante entra en relación con elementos que le hacen descubrir el mundo de los conocimientos, valores y la dimensión ética de los problemas; "que descubre la importancia de lo humano por encima de lo técnico-económico, de en ello se produce una educación del comportamiento a través de pauta de rigor, de apertura intelectual de capacidad de trabajo en equipo" (Rodríguez Izquierdo - Gavala, en Fernández,1993,).

Los indicadores serán respondidos mediante valores con: LIKERT

\section{ÍTEM}

1. Totalmente en desacuerdo

2. En desacuerdo

3. Ni de acuerdo, ni en desacuerdo

4. De acuerdo

5. Totalmente de acuerdo

\section{MARCO TEÓRICO}

\section{Marco filosófico o epistemológico Marco filosófico}

Nuestro estudio está basado en la concepción filosófica determinista, objetiva y realista, dado que, nuestro planteamiento parte de la realidad objetiva en lo tangible, como es la existencia de 7 universidades públicas y 26 universidades privadas en Lima metropolitana, que poseen una realidad inherente de responsabilidades en la formación de profesionales, los que asumen funciones de 
especialidad ante la Nación, nuestra búsqueda son las causales y consecuencias de la formación profesional en la especialidad de auditoría.

\section{Línea de Investigación.-}

Nuestro estudio se enmarca dentro de la línea: "Nuevas Técnicas y procedimientos de la moderna auditoría integrada, instrumento de evaluación y control de la calidad en la auditoría dirigida a la calidad del negocio", constituye como resultado de la currícula del Doctorado en Ciencias Contables y Empresariales de la Universidad Nacional Mayor de San Marcos".

\section{METODOLOGÍA}

\section{Tipo y diseño de investigación}

En esta parte procederemos a reconocer el ámbito epistemológico en la cual nos debemos desenvolver; desde el punto de vista:

Filosofía de la investigación. En concordancia con MARIO BUNGE (Epistemología, 1980, Editorial Ariel-Barcelona), el trabajo se enmarca dentro del conocimiento previo que presupone la investigación científica mencionada, en la que constata la validez, la existencia y cognoscibilidad del mundo externo; nuestro estudio estuvo dentro de la esfera técnico y moral al enfocar la "problemática”, así como netamente objetivo y realista; se estableció una metodología que permita lograr procedimientos pertinentes dirigidos por reglas que logren conocimiento objetivo que se puedan justificar teóricamente y empíricamente.

El enfoque de la investigación es del tipo: Abductivo creativo, porque parte de hechos explicados de tipo inductivo, que han permitido esclarecer una realidad (marco teórico); por lo tanto, considerada cierta, se debe lograr un nuevo caso explicativo y plausible, forma de cambio epistémico por medio del cual se pretende nueva posición de los hechos. La explicación que se pretende debe ser simplemente una sugerencia que deberá ser puesta en prueba antes de ponerse como creencia teórica.

Estrategias de Investigación.- Se encuadra en la experiencia laboral del autor y las teorías que fundamentan la especialidad de trabajo de auditoría.

Horizonte de tiempo.- Será de tipo transversal, porque se fundamentan en hechos varios, de un periodo de tiempo determinado.
Métodos de colección de datos.- Datos primarios/secundarios; entrevistas; observaciones.

Método de análisis de datos.- Descriptivos y cualitativos, dado que la evaluación se fundamenta en el logro de un diseño conceptual que permita formar profesionales dentro de un marco del conocimiento, experiencias y vivencias personales (aspectos que implican grados de subjetividad del ser humano como sujeto a tratar)

La concepción general del trabajo, se enmarcó:

Trasfondo filosófico.- Nos encontraremos dentro del contexto de demarcación, dado que, lo que se pretende es lograr una propuesta para un acuerdo o convención; ontológico, la labor se fundamenta en una realidad considerada material (objetiva), como gnoseológica, en el que se busca conocimiento (teoría del conocimiento); así mismo, racional conceptual y lógica, porque se expondrán presuposiciones de la investigación teórica en los campos de la ciencia y de la técnica; constituye la investigación acciones metodológicas, ya que la investigación parte de cuestionar y termina dentro del proceso, encontrando confirmaciones que han permitido llevar a cabo la presente investigación a realizar.

El aspecto formal.- Se encuadraría dentro de los alcances de tipo lógico para consistente, como una formalización de la dialéctica a racionalizar en el trabajo.

El cuerpo de conocimiento.- Estuvo dentro de la naturaleza y alcance de las Ciencias Sociales, dentro las cuales se encuentran las Ciencias administrativas; así como las Ciencias Contables; constituyendo el fondo del conocimiento: la persona humana (conocimientos, experiencias y vivencias) insertada en el contexto profesional (trabajo laboral), en la especialidad de Auditoría Administrativa/Contable.

Unidad de análisis.- Un eficiente trabajo de auditoría puede ser garantizado en la medida que el equipo de trabajo de auditoría conozca la operatividad y se apoyen en una metodología que norme su actuación.

Los niveles de productividad de las Instituciones en general podrán mejorar cuando las carencias y deficiencias sean detectadas a tiempo a través de una Auditoría Financiera y/o Administrativa oportuna.

La gestión administrativa en las empresas se basa en los objetivos, en los recursos y en el siste- 
ma de información implementado. Ello dependerá de la apropiada formación profesional de los auditores a cargo; es la INSTITUCIÓN UNIVERSITARIA responsable, a través de sus directivos a quienes se les entrevistó mediante los instrumentos pertinentes; por consiguiente es el sujeto en estudio, en la presente investigación.

Población de Estudio.- El universo poblacional está constituido por 33 Universidades pública y privadas en Lima metropolitana. Lo que significa el alcance en el estudio ( 7 públicas y 26 privadas ).

Tamaño de la muestra.- La muestra es la misma población, establecida como alcance en el estudio; por lo que no requerimos establecer cantidad alguna mediante fórmula. Dado que, al establecer mediante formula pre-establecida estadísticamente, la cantidad como elemento de muestra, nos estaría ofreciendo aproximadamente: 30 elementos a ser investigados; lo preocupante es la muestra, por ser pequeña dentro del ámbito No paramétrico de la estadística; aunque existen ventajas de aplicarse dichos métodos; en este caso, prevalece a opinión del investigador, que los métodos no paramétricos, tienden a perder información, porque datos numéricos exactos son reducidos frecuentemente a una formula cualitativa; y la pruebas no paramétricas no son tan eficientes como la pruebas paramétricas, de manera que con una prueba no paramétrica se necesita generalmente evidencia más fuerte.( así como una muestra más grande).

\section{Análisis e interpretación de la información.-}

El estudio se divide en dos fases:

EL PROCESO.- Constituido por labores realizadas por los profesores encargados de la formación universitaria, mediante cursos de la especialidad y otros que contribuyen al conocimiento, prácticas y formación ética/moral del alumno; Para ello, se ha utilizado: SPSS, como soporte de cálculo.

Así mismo, por cada pregunta de las entrevistas realizadas se consideró realizar también como un medio descriptivo: los respectivos estadísticos, cuyos comentarios se encontrarán al pie de los mismos.

EL ANÁLISIS DE LOS RESULTADOS.- Es el grado que se evalúa a través de los criterios y opiniones de los Directivos, quienes estiman sus resultados, como formadores de futuros profesionales cuyo contexto implica la resonancia de los conceptos de: CONOCIMIENTOS, EXPERIENCIAS, Y ETICO/ MORAL; así como estadísticos descriptivos.

Validación del instrumento de medición. Para la presente investigación se utilizó como instrumento de medición el paquete computarizado, SPSS (STATISTICAL PACKAGE SOCIAL SCIENCEPAQUETE ESTADÍSTICO PARA LAS CIENCIAS SOCIALES), aplicados indistintamente a cada uno de los personajes.

\section{RESULTADOS}

\begin{tabular}{ccrr}
\multicolumn{4}{c}{ Análisis de fiabilidad } \\
Escala: TODAS LAS VARIABLES \\
Resumen del procesamiento de los casos \\
\hline Válidos & $\mathrm{N}$ & $\%$ \\
\hline \multirow{3}{*}{ Casos } & Excluidos(a) & 0 & 100.0 \\
& Total & 66 & .0 \\
Fuente primaria. & & &
\end{tabular}

a. Eliminación por lista basada en todas las variables del procedimiento.

Estadísticos de fiabilidad

\begin{tabular}{cc}
\hline Alfa de Cronbach & N de elementos \\
\hline 0.891 & 14
\end{tabular}

Uno de los coeficientes más comunes para calcular la fiabilidad es el Alpha de Cronbach (Cronbach, 1951) que se orienta hacia la consistencia interna de una prueba. El modelo asume que la escala compuesta por elementos homogéneos aleatoriamente seleccionados de la población de los posibles elementos que miden la misma característica; en nuestro estudio, el 1 caso de la formación profesional de los auditores y sus variables tratadas, existe una fiabilidad de 0.891 , que nos indica una alta homogeneidad y equivalencia de respuesta a todos los ítems a la vez y para todos los encuestados dentro de 14 elementos.

\section{DISCUSIÓN}

En este estudio se abordó la representación gráfica de los resultados, constatando su utilidad en el proceso de análisis estadístico y la presentación de datos. Se describirán los distintos tipos de gráficos que podemos utilizar y su correspondencia con las distintas etapas del proceso de análisis. 
Un primer paso consistió en presentar esa información de forma que se pueda visualizar de una manera más sistemática y resumida. Los datos que nos interesan dependen, en cada caso, del tipo de variables que estamos manejando.

\begin{tabular}{lcc}
\multicolumn{3}{c}{ Formación } \\
\hline & Frecuencia & Porcentaje \\
\hline en desacuerdo & 7 & 10,6 \\
ni de acuerdo, ni en desacuerdo & 12 & 18,2 \\
de acuerdo & 23 & 34,8 \\
totalmente de acuerdo & 24 & 36,4 \\
\hline \multicolumn{1}{c}{ Total } & 66 & 100,0 \\
\hline
\end{tabular}

Respecto a la necesidad de realizar mejoras en la gestión académica universitaria inherente en la formación de los auditores, el 34,8\% de la muestra se encuentra de acuerdo con ello y el $36,4 \%$ totalmente de acuerdo.

\section{Mejoramiento continuo (conocimientos)}

El auditor debe tener estudios adicionales que le permitan tener nivel para intervenir, aparte de ser administrador o contador.

\begin{tabular}{lcc}
\hline & Frecuencia & Porcentaje \\
\hline de acuerdo & 35 & 53,0 \\
\hline totalmente de acuerdo & 31 & 47,0 \\
\hline Total & 66 & 100,0
\end{tabular}

Respecto a la necesidad de que el auditor posea estudios relacionados con la informática, comunicación, administración pública, psicología, pedagogía, ingeniería industrial y de sistemas, derecho, entre otros, la muestra se encuentra de acuerdo en un $53 \%$ y totalmente de acuerdo $47 \%$.

Es necesario instruir a los auditores durante su formación universitaria en temas adicionales especializados en materias de auditoría.

\begin{tabular}{lcc}
\hline & Frecuencia & Porcentaje \\
\hline de acuerdo & 32 & 48,5 \\
\hline totalmente deacuerdo & 34 & 51,5 \\
\hline Total & 66 & 100,0 \\
\hline
\end{tabular}

Con respecto a la necesidad de que los auditores se instruyan en aspectos especializados de materia tributaria, en foros, conferencias, talleres o seminarios, el $48,5 \%$ de los entrevistados se muestra de acuerdo y $51,5 \%$ totalmente de acuerdo.
Los conocimientos resultantes de la implementación de auditores en diferentes instituciones en materias especializadas, permitirán conocimientos idóneos para un mejor logro profesional de los mismos.

\begin{tabular}{lcc}
\hline & Frecuencia & Porcentaje \\
\hline \multicolumn{1}{c}{ de acuerdo } & 39 & 59,1 \\
\hline totalmente de acuerdo & 27 & 40,9 \\
\hline Total & 66 & 100,0 \\
\hline
\end{tabular}

En este caso se le mostró a cada uno de los funcionarios entrevistados una lista de temas como logística, empowerment, liderazgo, series de tiempo, sistemas y procedimientos, costos, presupuestos, benchmarking, modelos de inventario, entre otros. El 59,1\% de la muestra manifiesta su acuerdo y el $40,9 \%$ indica estar totalmente de acuerdo.

Durante la formación académica universitaria el auditor debe saber operar equipos de cómputo, así como el manejo de sistemas de información a nivel estructural.

\begin{tabular}{lcc}
\hline & Frecuencia & Porcentaje \\
\hline \multicolumn{1}{c}{ de acuerdo } & 40 & 60,6 \\
\hline totalmente de acuerdo & 26 & 39,4 \\
\hline Total & 66 & 100,0 \\
\hline
\end{tabular}

Respecto a esta afirmación, $60,6 \%$ de la muestra se manifiesta de acuerdo y $39,4 \%$ totalmente de acuerdo.

\section{Experiencia}

El auditor adquiere dominio con el día a día en base a las iniciativas o programas orientados a la producción de resultados para la organización.

\begin{tabular}{lcc}
\hline & Frecuencia & Porcentaje \\
\hline en desacuerdo & 1 & 1,5 \\
\hline nideacuerdo, niendesacuerdo & 9 & 13,6 \\
\hline de acuerdo & 40 & 60,6 \\
\hline totalmente de acuerdo & 16 & 24,2 \\
\hline Total & 66 & 100,0 \\
\hline
\end{tabular}

Con respecto a esta afirmación el 60,6\% de los funcionarios entrevistados se muestra de acuerdo, $24,2 \%$ totalmente de acuerdo. Mientras que 13,6\% se muestra indiferente al respecto (ni de acuerdo ni en desacuerdo) y sólo 1,5\% en desacuerdo.

La competencia en las áreas adjetivas de la organización se deriva de la aplicación continua de las medidas de orden administrativo. 


\begin{tabular}{lcc}
\hline & Frecuencia & Porcentaje \\
\hline en desacuerdo & 2 & 3,0 \\
\hline nideacuerdo,niendesacuerdo & 8 & 12,1 \\
\hline de acuerdo & 40 & 60,6 \\
\hline totalmente de acuerdo & 16 & 24,2 \\
\hline Total & 66 & 100,0 \\
\hline
\end{tabular}

Con respecto a esta afirmación, el 60,6 de los entrevistados señala estar de acuerdo con la afirmación, 24,2\% está totalmente de acuerdo; $12,1 \%$ no está ni de acuerdo ni en desacuerdo y $3 \%$ en desacuerdo.

Los esfuerzos anteriores permiten un mejor entendimiento del proceso de aplicación de una auditoría.

\begin{tabular}{lcc}
\hline & Frecuencia & Porcentaje \\
\hline en desacuerdo & 2 & 3,0 \\
\hline nideacuerdo,niendesacuerdo & 8 & 12,1 \\
\hline de acuerdo & 40 & 60,6 \\
\hline totalmente de acuerdo & 16 & 24,2 \\
\hline Total & 66 & 100,0
\end{tabular}

En este caso 60,6 de los entrevistados señala estar de acuerdo con la afirmación, $24,2 \%$ está totalmente de acuerdo; $12,1 \%$ no está ni de acuerdo ni en desacuerdo y $3 \%$ en desacuerdo.

El desarrollo de casos prácticos permite coadyuvar a la percepción de la naturaleza, alcance y condiciones de las técnicas que se utilizan para auditar una organización.

\begin{tabular}{lcc}
\hline & Frecuencia & Porcentaje \\
\hline en desacuerdo & 2 & 3,0 \\
\hline nideacuerdo, niendesacuerdo & 8 & 12,1 \\
\hline de acuerdo & 40 & 60,6 \\
\hline totalmente de acuerdo & 16 & 24,2 \\
\hline Total & 66 & 100,0
\end{tabular}

En relación a esta afirmación, 60,6 de los entrevistados señala estar de acuerdo con la afirmación, $24,2 \%$ está totalmente de acuerdo; $12,1 \%$ no está ni de acuerdo ni en desacuerdo y $3 \%$ en desacuerdo.

Las experiencias derivadas de estudios organizacionales de otra naturaleza permiten apreciaciones de los requerimientos necesarios para instrumentar una auditoría.

\begin{tabular}{lcc}
\hline & Frecuencia & Porcentaje \\
\hline en desacuerdo & 2 & 3,0 \\
\hline nideacuerdo,niendesacuerdo & 8 & 12,1 \\
\hline de acuerdo & 40 & 60,6 \\
\hline totalmente de acuerdo & 16 & 24,2 \\
\hline Total & 66 & 100,0 \\
\hline
\end{tabular}

En relación a este aspecto, el 60,6 de los entrevistados señala estar de acuerdo con la afirmación, $24,2 \%$ está totalmente de acuerdo; $12,1 \%$ no está ni de acuerdo ni en desacuerdo y $3 \%$ en desacuerdo.

Los atributos personales permiten un dominio de las áreas de conocimiento administrativo, métodos de trabajo, técnicas de estudio o influencia en la organización.

\begin{tabular}{lcc}
\hline & Frecuencia & Porcentaje \\
\hline en desacuerdo & 2 & 3,0 \\
\hline nideacuerdo,niendesacuerdo & 8 & 12,1 \\
\hline de acuerdo & 40 & 60,6 \\
\hline totalmente de acuerdo & 16 & 24,2 \\
\hline Total & 66 & 100,0 \\
\hline
\end{tabular}

Los entrevistados tuvieron acceso a una relación de atributos como liderazgo formal, liderazgo informal, inteligencia, sentido común e interés por aprender. Al respecto, el 60,6 de los entrevistados señala estar de acuerdo con la afirmación, 24,2\% está totalmente de acuerdo; $12,1 \%$ no está ni de acuerdo ni en desacuerdo y $3 \%$ en desacuerdo.

\section{Ética / Moral}

Es necesario fomentar los valores morales (éticos) en las instituciones educativas, a través de cualquier asignatura.

\begin{tabular}{lcc}
\hline & Frecuencia & Porcentaje \\
\hline de acuerdo & 35 & 53,0 \\
\hline totalmente de acuerdo & 31 & 47,0 \\
\hline Total & 66 & 100,0 \\
\hline
\end{tabular}

Los entrevistados manifestaron en un $52 \%$ su acuerdo con esta afirmación, mientras que $47 \%$ señaló su total acuerdo.

Se justifica la introducción en las currículas lectivas de las universidades, no solo del curso de Ética, sino el esfuerzo académico por crear y prestigiar una cátedra seria de Educación Moral

\begin{tabular}{|l|c|c|}
\hline & Frecuencia & Porcentaje \\
\hline de acuerdo & 32 & 48,5 \\
\hline totalmente de acuerdo & 34 & 51,5 \\
\hline Total & 66 & 100,0 \\
\hline
\end{tabular}

Con respecto a esta afirmación, el $48,5 \%$ manifestó su acuerdo, mientras que $51,5 \%$ señaló su total acuerdo.

La formación ética del profesor es un factor determinante en su actividad docente para lograr formación integral del auditor. 


\begin{tabular}{lcc}
\hline & Frecuencia & Porcentaje \\
\hline de acuerdo & 39 & 59,1 \\
\hline totalmentedeacuerdo & 27 & 40,9 \\
\hline Total & 66 & 100,0 \\
\hline
\end{tabular}

En relación a esta afirmación, el 59,1\% manifestó su acuerdo, mientras que 40,9\% señaló su total acuerdo.

\subsection{Prueba de hipótesis}

Estimamos que los análisis e interpretación de las variables nos permiten justificar nuestra hipótesis, permitiendo que el nivel de la auditoría y la calidad de su resultado conocimiento,experiencia, ético / moral- en tareas de evaluación en las organizaciones.

\subsection{Presentación de resultados}

\section{Relación entre Formación y Mejoramiento continuo (Conocimientos)}

En este caso se ha utilizado la correlación de rangos de Spearman, en tanto la medición de las variables se realizó mediante escala ordinal (Likert). Los valores de este tipo de correlación oscilan entre -1 y 1 .-

\section{Correlaciones}

\begin{tabular}{|c|c|c|c|c|}
\hline & & & Formació & nocimiento \\
\hline \multirow{6}{*}{$\begin{array}{c}\text { Rho de } \\
\text { Spearman }\end{array}$} & \multirow{3}{*}{ Formación } & $\begin{array}{c}\text { Coeficiente de } \\
\text { correlación }\end{array}$ & 1,000 &, $794 * *$ \\
\hline & & Sig. (bilateral) & . & ,000 \\
\hline & & $\mathrm{N}$ & 66 & 66 \\
\hline & \multirow{3}{*}{$\begin{array}{l}\text { Conoci- } \\
\text { miento }\end{array}$} & $\begin{array}{c}\text { Coeficiente de } \\
\text { correlación }\end{array}$ &, $794^{* *}$ & 1,000 \\
\hline & & Sig. (bilateral) & ,000 & . \\
\hline & & $\mathrm{N}$ & 66 & 66 \\
\hline
\end{tabular}

** La correlación es significativa al nivel 0,01 (bilateral).

Para la relación entre formación y conocimientos se ha obtenido un coeficiente de correlación igual a 0.794 , el mismo que indica asociación positiva y directa entre ambas variables, de lo que se desprende que mejores niveles de conocimiento se corresponden con mejores niveles de formación. La asociación es significativa con un 95\% de confianza. Ello se ilustra con el diagrama de dispersión mostrado a continuación.

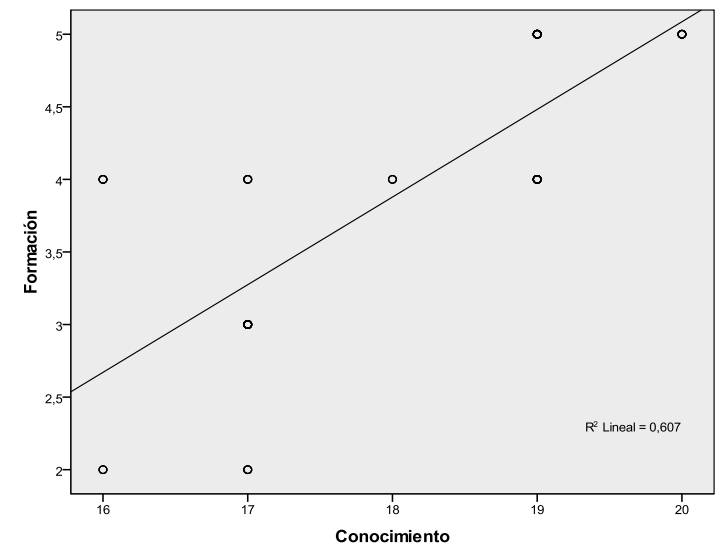

Relación entre Formación y Experiencia Correlaciones

\begin{tabular}{cccc}
\hline & \multicolumn{3}{c}{ Formación Experiencia } \\
\hline \multirow{2}{*}{$\begin{array}{c}\text { Rho de } \\
\text { Spearman }\end{array}$} & $\begin{array}{c}\text { Coeficientede } \\
\text { correlación }\end{array}$ & 1,000 &, $968^{* *}$ \\
\cline { 2 - 4 } & Experiencia (bilateral) &. &, 000 \\
\cline { 2 - 4 } & $\begin{array}{c}\text { Coeficientede } \\
\text { correlación }\end{array}$ &, $968^{* *}$ & 1,000 \\
\cline { 2 - 4 } & Sig. (bilateral) &, 000 &. \\
\cline { 2 - 4 } & $\mathrm{N}$ & 66 & 66 \\
\hline
\end{tabular}

**. La correlación es significativa al nivel 0,01 (bilateral).

La correlación para las variables formación y experiencia es de 0.968 , el mismo que denota una asociación muy fuerte, positiva y directa. Ello significa que mejores niveles de experiencia se corresponden con mejores niveles de formación, esto es, que la experiencia es un factor importante en la formación del auditor.

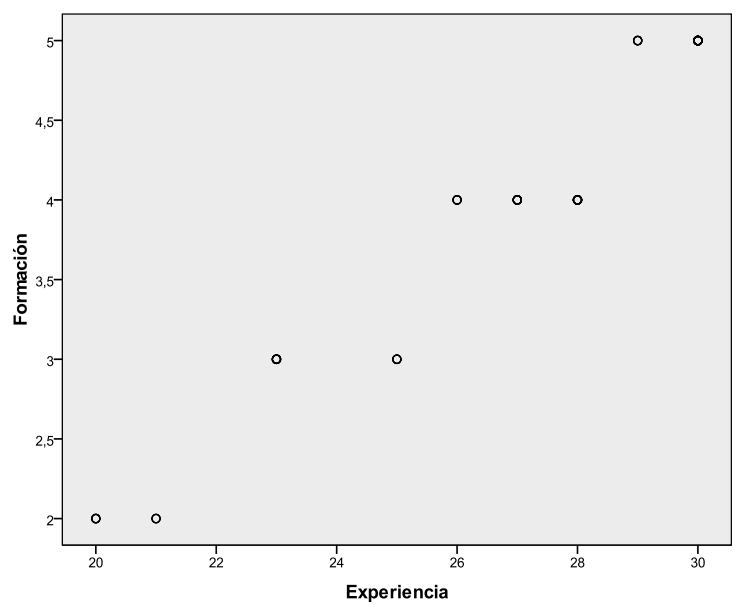




\section{Relación entre Formación y Ética Correlaciones}

\begin{tabular}{cccc}
\hline & \multicolumn{3}{c}{ Formación EticayMoral } \\
\hline \multirow{3}{*}{$\begin{array}{c}\text { Rho de } \\
\text { Spearman }\end{array}$} & $\begin{array}{c}\text { Coeficiente de } \\
\text { correlación }\end{array}$ & 1,000 &, $794^{* *}$ \\
\cline { 2 - 4 } & Etica y Moral (bilateral) &. &, 000 \\
\cline { 2 - 4 } & $\begin{array}{c}\text { Coeficiente de } \\
\text { correlación }\end{array}$ &, $794^{* *}$ & 1,000 \\
\cline { 2 - 4 } & Sig. (bilateral) &, 000 &. \\
\cline { 2 - 4 } & $\mathrm{N}$ & 66 & 66 \\
\hline
\end{tabular}

**. La correlación es significativa al nivel 0,01 (bilateral).

El coeficiente de correlación de Spearman para la relación entre formación y los aspectos de ética y moral es de 0.794 , valor que indica asociación positiva y directa. A mayores niveles de ética y moral le corresponden mayores niveles de formación.

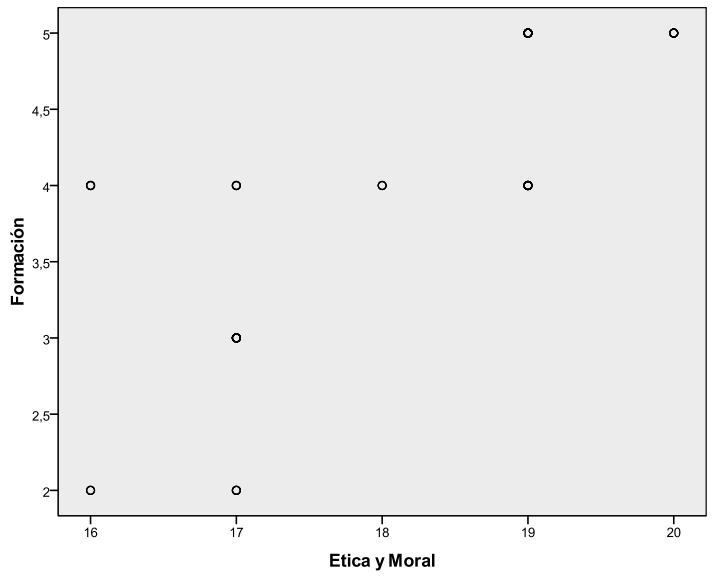

\section{CONCLUSIONES}

1. Se estima que formación profesional sistematizada son todos aquellos estudios y aprendizajes encaminados a la inserción, reinserción y actualización laboral, cuyo objetivo principal es aumentar, adecuación del conocimiento y habilidades de los actuales y futuros auditores administrativos /contables a lo largo de toda la vida.

El intelecto y en el contexto donde nos desarrollamos se generan ideas, pero estas tienen sentido y se enriquecen sólo en la experiencia. Y en la profesión como auditor, la experiencia surge, se transforma y se reformula durante el ejercicio práctico, dentro de un marco ético/moral, para elaborar planes de trabajo, proyectos de desarrollo, programas o sistemas, así como modelos de evaluación.
2. La formación profesional continua, la experiencia, como la ética/moral orientada al colectivo de auditores administrativos/ contables en actividad, cuyo objetivo fundamental es la adquisición de mayores competencias que le permitan una actualización permanente en la función que desempeñan, lo que en definitiva se resume como un aumento de su diligencia en sus labores. Redundando en el nivel de la especialidad en auditoría y la calidad que dependen del profesionalismo y sensibilidad del auditor, así como de su compromiso en las actividades y/o procesos que evalúa.

3. Los conocimientos, la experiencia, así como lo ético/moral, son los elementos fundamentales en las labores del Auditor, se encuentran dentro del propio ser del trabajador, haciendo con ello posible la apropiación de la independencia de criterio que le asiste como persona en funciones. La falta de estas características como elementos fundamentales del proceso de trabajo del auditor, hace posible la continuación de las relaciones existentes como las limitaciones al alcance de los trabajos y/o presiones de poder. Son condiciones que desfavorecen el desarrollo integral del auditor y sus objetivos laborales.

\section{RECOMENDACIONES}

1. EN CUANTO A LAS UNIVERSIDADES.- Deberán ser responsables de formar auditores administrativos/contables deben orientar sistemáticamente a la especialización profesional de la actividad concreta, en un ámbito intelectual restringido a las necesidades específicas de la función, en el contexto integral del conocimiento, experiencia y ética/moral en la persona como capital humano.

Las universidades, en niveles interfacultativos, deben crear un sistema inherente al proceso de enseñanza-aprendizaje, en forma integral y continua, que estimule y desarrolle las capacidades, aptitudes, así como la creatividad, que permita desarrollar en los futuros profesionales, características necesarias en la personalidad del auditor administrativo / contable, permitiendo en lo académico, desempeñar un papel relevante, toda vez que puedan aportar elementos de 
juicio por su conocimiento y experiencia en las áreas de las organizaciones; en los aspectos del aprendizaje y experiencia complementarias, puedan tener participación activa en los eventos de formación, capacitación, desarrollo y actualización; como seminarios, congresos, paneles, encuentros, cursos, talleres, foros o conferencias; así como marcos de experiencia empírica; conocimientos y experiencias resultantes de implementación en su formación de auditorías en diferentes instituciones.

La especialización profesional daría lugar al conocimiento de asignaturas o disciplinas científicas y humanistas que justifique su propia labor de integración al momento de evaluar funcionalmente una organización. La idea es el aumento de la capacidad de profundizar en el conocimiento, sin perder la perspectiva de conjunto (holístico o integrador).

La sociedad está en continuo cambio, lo que ayer facilitaba el acceso al mercado laboral, puede que mañana esté obsoleto. Los jóvenes con formación superior se ven obligados a trabajar en situaciones desfavorables funcionalmente. Por ello, es importante formar alumnos para ser auditores administrativos/ contables dentro de un contexto sistémico e integrador al mundo empresarial que lo rodea y, hacer ver lo importante de aprender a emprender con calidad.

Por ello la presente investigación propone cinco indicadores en los se deberían desarrollar los temas directamente relacionados con la formación profesional del auditor, en especial con la práctica y la administración de sus recursos.

- Características de la profesión del auditor para el cambio y su desarrollo, en la que debe destacar las tareas de capacitación y actualización de los profesionales en la materia de auditoría.

- La planificación y el desarrollo institucional, en el que debe presentar la propuesta para diseñar planes institucionales, resultando en programas académicos y de investigación de instituciones.

- Se debe elaborar proyectos de formación, inmersos en la tecnología que coadyuve como referencia para el profesor o formadores, el estudiante, el administrador o el responsable del monitoreo de la gestión.

- Se debe poner énfasis en la docencia en el aula, definiendo e incluyendo tecnologías para diseñar programas de curso y proyectos de investigación o para elaboración de propuestas curriculares de uso práctico y flexible

- La evaluación de los programas de desarrollo de la formación profesional del auditor. Se debe proponer modelos de evaluación de los proyectos académicos, como eventos de investigación o de difusión de la cultura científica, tecnológica y humanística; y por supuesto, no debe faltar propuestas para evaluar los procesos de enseñanza y aprendizaje

2. En cuanto al Estado.- Es necesario de Políticas Públicas de formación profesional en la especialidad profesional de Auditoría que permita, además de perspectivas del conocimiento, se requiera la voluntad del Estado el de asumir integralmente el problema, impulsando conocimiento y experiencias.

Con el apoyo de la Escuela Nacional de Control de la Contraloría General de la República, la Superintendencia Nacional de Administración Tributaria, los Ministerios, así como los Gobiernos Locales y otros, debieran colaborar en la formación integral de los estudiantes de auditoría; dado que también son parte de la problemática funcional del auditor, ya que requieren de éste, un profesional que permita en ejercicio de sus funciones, del control, fiscalización o revisión de manera profesional, independiente y confiable. Si ese es su requerimiento, deben contribuir en forma obligada en aceptarlos en sus entidades como Estudiantes Internos, ganando las experiencias necesarias integrando las Comisiones de Auditorías; en el caso de la Escuela Nacional de Control de la Contraloría, participando en los diversos cursos de la especialización; constituyendo, parte de la hoja de vida del estudiante que la universidad debiera monitorear, consideraciones muy similares al SERVICIO RURAL Y URBANO MARGINAL EN SALUD (SERUM). 
3. En cuanto a las Entidades Privadas:

Los Colegios Profesionales, Sociedades de Auditoría, Empresas Comerciales e Industriales, en forma similar debieran estar sujetos a la aceptación de estudiantes practicantes dentro de sus órganos de Control Interno (Auditoría Interna).

Lo expresado constituiría un enfoque sistémico que coadyuve en la formación profesional del auditor como capital humano, prácticas objetivas y calificadas por dichas instituciones; previa a la titulación correspondiente (dado que sería un mérito obligatorio), a la Especialización de Auditoría Administrativa/contable, dentro del contexto profesional.

\section{REFERENCIAS BIBLIOGRÁFICAS Bibliografía Básica}

Alvira. Metodología de la evaluación de programas. Cuaderno Metodológico. №. 2, Madrid, 1991. p-33

Angulo. Teoría y Desarrollo del Curriculum / José Félix Angulo, Nieves Blanco. Granada: Ed. Algibe, 1994 . p 25.

Añorga Morales, Julia. Teoría de los Sistemas de Superación. La Habana. [s.n.], 1995 .--p 9.

Arnal, Justo. del Rincón, Investigación Educativa. Fundamentos y metodología. Editorial Labor. Barcelona, 1994.-- 79 p

Barnes, Tony. Como lograr un liderazgo exitoso "kaisen”. Editorial Mc Graw Hill. 2001. México.

Berman, P. Federal Programs Supporting Education Change. Vol IV .1990.-- p.10

Bunge Mario (Epistemología -1980-Editorial Ariel31-Barcelona)

Cronbach L (1951) Coeffient Alpha and the Internal Structure of Test. Psycometrika (setiembre) pp 297-334.

Cruz Cardona, Víctor. Guía de Autoevaluación/ Víctor Cruz Cardona.-- España: AVIP, 1995 -- p 36.

Díaz Santos, Sep 3, 203-368 pg. Estadística Aplicada: Una visión instrumental.

Félix Armando Rivera León. 2007. Análisis del trabajo de las prácticas de auditoria de las empresas de servicios públicos en Lima metropolitana, 2000-2004./ (Tesis de MaestríaUNMSM -Lima-Perú

Fernández Arenas, José Antonio, (1996),'La Auditoria Administrativa", Editorial Jus s.a. -Mexico.

Fernández Barcala M. (2000),”Validación de Serqual como instrumento de medida de calidad de servicio bancario. Revista Europea de Dirección y Economía de la Empresa, volumen 9, No. Pp 57-70.

Ferreiro Grovie, Ramón. Paradigma Científico, Tendencias contemporáneas y formación posgraduada de especialistas en Ciencias de la Educación. Cuba Instituto Central de Ciencias Pedagógicas. I Junta Consultiva sobre Postgrado en Iberoamérica. Dic. 1992.

García, Miriam Luci. Trabajo presentado en la Junta Consultiva de Posgrado en Iberoamérica/ Miriam Luci García.-- La Habana.-- Elaborado en el ISP “ .Enrique José Varona”, 1962.--66p.

Idalberto Chiavenato. Gestión del talento humano. Editorial Mc Graw Hill. 2002. México.

Instituto de Ingenieros Industriales. Mas allá de la Reingeniería. Editorial CECSA. Primera Edición en 1995. México. 1995. 245 pag.

Johannsson Henry J., McHUGH Patrick Y Otros. Reingeniería de Procesos de Negocios. Editorial LIMUSA.. México. 1994. 268 pag.

Koufman, Rogers y Adriana Vergara. Determinación de necesidades de donde vienen los objetivos. Revista Tecnología Educativa. (Buenos Aires) 9,(2): p 259. 1984.

Koufman, Roger. Planificación de Sistemas Educativos. Editorial Trillas, 1980.-- de Sistemas: Un enfoque prospectivo. / Felipe Lara Rosano. -- México : ( s n), 1990.-- p 4.

Leiva González, David. Reflexiones sobre capacitación docente. David Leiva. Gonzáles.-- México: Ed. Consejo Coordinador de Sistemas Abiertos, 1981.--p.7.

Mac Donald, B. Changing the curriculum.-- p 4-5.

Merten, 2005, en su Research and evaluation in Education and Psychology: Ingrating Diversity with quantitative, qualitative and mixedmethods(2a. ed.). Thousand Oaks: Sage. 
Metodología General de la Evaluación de impacto ambiental.--Ecuador: [s.a.], 1994-p 6.

Miguel Díaz, Mario de. Evaluación y desarrollo profesional docente / Mario de Miguel Díaz .-- España: Ed. Universidad de Oviedo, 1993.-- p.19.

Morales, Víctor. Hacia los Sistemas Nacionales de estudios de Avanzadas en América y el Caribe. Venezuela: Elaborado en la Universidad Central de Venezuela, 1990.--p 14.

Morris Daniel \& Brandon Joel. Reingeniería. Editorial Mc Graw Hill. Colombia. 1994. 298 pág.

Noguez, Antonio. Sistematización de la Enseñanza, determinación necesidades educativas / Antonio Noguez, David Leyva.- México. Ed. Instituto Latinoamericano de la Comunicación Educativa, 1992 .-- p.50.

Pere Escorsa Castells, Jaume Valls Pasola. 2001. Tecnología e innovación en la empresa dirección y gestión. Ediciones UPC. España.

Peter Checkland, Jim Scholes. La metodología de sistemas suaves en acción. Editorial Limusa. México. 1994

Raymond L. Manganelli, Mark M. Klein. Cómo hacer Reingeniería. Grupo Editorial Norma. 1995. Colombia.

Raymond Mcleod Jr. Sistemas de Información Gerencial. 7edición. Pearson Educación de México.1998

Resúmenes. III Junta Consultiva del Posgrado Iberoamérica Ciudad Habana. Cuba.--1996. -- p.33

Roger, E. Comunication and innovation: A cross cultural approach. (2nd edition).(s.l:s.n.s.a) -- p.19

Rodriguez Izquierdo y Gavala (1993) "Como ocupan los universitarios almeriences su tiempo libre", Universidad de Almería - España

Rodriguez Mejia , José Carlos (Plan de Desarrollo Humano Loca....(Municipalidad de Hojancha"- Costa Rica.

Rutman, L. Evaluación de programas. En : Castellano Quintero, S. Apuntes bibliográficos para una Evaluación de Proyectos Educativos y Educación Comparada. Ecuador, Editorial Grafioffset, 1997. p-27.

Sapag Chain, N. Preparación y evaluación de Pro- yectos. Segunda Edición. 1995.

Scriven, M. Modelo de Evaluación. En: Castellano Quintero, S. Apuntes bibliográficos para una Evaluación de Proyectos Educativos y Educación Comparada Ecuador, Editorial Grafioffset, 1997. p-41.

Selltiz, C.JOhoda ,M.Deutsch,M. y Cook,S. W. (1980).Métodos de investigación en la relaciones sociales (9a. Ede). Madrid: Rialp.

Shadish, W. Modelo de Evaluación. En : Castellano Quintero, S. Apuntes bibliográficos para una Evaluación de Proyectos Educativos y Educación Comparada. Ecuador, Editorial Grafioffset, 1997. p-27.

Stephan Konz. Diseño de Sistemas de Trabajo. Editorial Limusa SA. 1999. México.

Stephen Robbins. 1999. Comportamiento organizacional. Editorial Prentice Hall. México.

Stufflebeam L, Daniel. Evaluación sistemática: Guía téorica y práctica. Daniel L. Stufflebeam y L.Shenkfied.-- Barcelona: Editorial Paidós, 1995.-- p 67.

Tagliacarne, Guillermo. Investigación y Análisis de mercado. Editorial Reverte. 1978.

Tyler, R. Modelo de Evaluación. En Castellano Quintero, S. Apuntes bibliográficos para una Evaluación de Proyectos Educativos y Educación Comparada. Ecuador, Editorial Grafioffset, 1997. p-34.

Visauta (1998) Tesis: “Gestión Orientada al Valor del Usuari@

\section{Otras referencias}

Ackoff. EI ENFOQUE DE SISTEMAS: TEORÍA GENERAL DE SISTEMAS ... a los enfoques analítico-mecánico y a los de la teoría general de sistemas. Esta ..... "un sistema abstracto es aquel en que lodos sus ele- ... www.unazulia.com/archivos/306/Lectura3.2.

Bunk, G. P. La transmisión de las competencias en la formación y perfeccionamiento profesionales en la RFA. Revista CEDEFOP No. 1. 1994.

http://www.ilo.org/public/spanish/region/ ampro/cinterfor/temas/complab/xxxx/esp/i/ index.htm\# 1

Carlos Monserrate (Director general de RB Tech- 
nology.net.). El Genoma del Talento: Hacia un modelo de ingeniería de Gestión del Talento. Revista: Capital Humano. № 170, octubre de 2003. http://www.capitalhumano.es

Coeficiente de correlación de Spearman - Wikipedia, la ...

Una generalización del coeficiente de Spearman es útil en la situación en la cual hay tres o más ...

es.wikipedia.org/.../Coeficiente_de_correlación_de_Spearman-

CONOCER. La normalización y certificación de competencia laboral: Medio para incrementar la productividad de las empresas. Presentación en Power Point. Marzo de 1997.

http://www.ilo.org/public/spanish/region/ ampro/cinterfor/temas/complab/xxxx/esp/i/ index.htm\# 1

Deyanira Meza Martell

El factor trabajo y la teoría de capital humano - 25 Abril 2000

http://www-csc.mty.itesm.mx/cgi-bin/csc/ HN sc214 abr2000/get/tema1/1.html

Ducci, María Angélica. El enfoque de competencia laboral en la perspectiva internacional. En: Formación basada en competencia laboral. Cinterfor/OIT. Montevideo. 1997.

http://www.ilo.org/public/spanish/region/ ampro/cinterfor/temas/complab/xxxx/esp/i/ index.htm\# 1

Gonczi, Andrew; Athanasou, James. Instrumentación de la educación basada en competencias. Perspectiva de la teoría y la práctica en Australia. Ed. Limusa. 1996

http://www.ilo.org/public/spanish/region/ ampro/cinterfor/temas/complab/xxxx/esp/i/ index.htm\# 1

Ibarra, Agustín. El Sistema Normalizado de Competencia Laboral. En: Competencia laboral y educación basada en normas de competencia. SEP, CONOCER, CONALEP. 1996.

http://www.ilo.org/public/spanish/region/ ampro/cinterfor/temas/complab/xxxx/esp/ ii/index.htm

INEM. Metodología para la ordenación de la formación profesional ocupacional. Sub- dirección general de gestión de formación ocupacional. Madrid. 1995.

http://www.ilo.org/public/spanish/region/ ampro/cinterfor/temas/complab/xxxx/esp/i/ index.htm\# 1

JACKSON. Didáctica en la Educación -SER SOCIAL Sistema de ideas, sentimientos y hábitos que expresa el grupo. ... Desde la perspectiva de la escuela como transmisora crítica de cultura reclaman un modelo de Diseño .... ..... proporcionan un poder heurístico ... html.rincondelvago.com/didactica-en-laeducacion.html

James G. Miller, "Living Systems: Basic Concepts", ORIGENES DE LA TEORIA DE SISTEMAS La teoría general de ... formulaciones conceptuales que puedan crear condiciones de aplicación en la ... b) Esa integración parece orientarse rumbo a una teoría de sistemas. ... circuito eléctrico, un computador o un ser vivo pueden ser visualizados como sistemas. ...

Jay Forrester. Teoría General De Sistemas Y dice: quot;...el modelo de equilibrio es aplicable a tipos de sistemas que se ...En el libro Industrial Dynamics, se recogen los planteamientos ..... mostrada representa al modelo de sistema viable(msv) de una organización, ... www.slideshare.net/.../ teoria-general-de-sistemas

Jérôme Gleizes .traduction castillane de Le capital humain, Multitudes 2 : mai 2000, Majeure : nouvelle économie politique, Notions

http://multitudes.samizdat.net/article. php3?id_article $=312$

Ludwig Von Bertalanffy. Capítulo 3: La Teoría General de Sistemas 1925 - 1926 - Teoría General de. Sistemas. • 1929, 1932 - Cannon con la Homeostasis. • 1947 - Von Newman y Morgenstern con La ... www. angelfire.com/planet/.../teoría gral sistemas_bertanlanffy

OIT. Formación profesional. Glosario de términos escogidos. Ginebra. 1993.

http://www.ilo.org/public/spanish/region/ ampro/cinterfor/temas/complab/xxxx/esp/i/ index.htm\# 1

Parasuraman y Berry Behavioral Science, 10 jul. ... www.eubca.edu.uy/materiales/...de.../ 
teoria_de_sistemas. SERVQUAL - Evaluación de la Calidad del Servicio - Aiteco ...El SERVQUAL es un instrumento, en forma de cuestionario, elaborado por Zeithaml, cuyo propósito es evaluar la calidad de servicio www.aiteco.com/servqual.htm

Peter Checkland. METODOLOGIA DE SISTEMAS BLANDOS 1 Ago 2009 ... EXPLICACIÓN La Metodología de sistemas blandos (SSM por sus siglas en inglés) es una técnica cualitativa que se ... www.scribd.com/.../ METODOLOGIA-DE-SISTEMAS-BLANDOS -
Spearman Charles. En estadística, el coeficiente de correlación, es una medida de la correlación (la asociación o interdependencia) entre dos variables.

Your people are your advantage. Let's talk about their potential.

http://www.arthurandersen.com/WebSite. nsf/Content/MarketOfferingsHumanCapita l?OpenDocument 